Revista Eletrônica de Ciência Administrativa (RECADM) - ISSN 1677-7387

Faculdade Cenecista de Campo Largo - Coordenação do Curso de Administração

v. 3, n. 1, maio/2004 - http://revistas.facecla.com.br/index.php/recadm/

\title{
ESTUDIO DE LA CARTERA DE AVALES DE LAS SOCIEDADES DE GARANTÍA RECÍPROCA ESPAÑOLAS EN 2002
}

\author{
Carmen Pérez López \\ Departamento de Economía Financiera \\ Universidad de Sevilla
}

\section{RESUMEN}

Nos centramos en este artículo en el estudio de los avales concedidos por las diferentes Sociedades de Garantía Recíproca, SGR, que operan en España; primero de una forma cuantitativa, analizando la evolución de la variable Riesgo Vivo, en el periodo 1997-2002. Posteriormente realizaremos un estudio cualitativo de los avales vigentes, atendiendo a siete perspectivas distintas: sectores de actividad, tamaño de las empresas, finalidad de las operaciones, plazo de amortización, importe medio, entidades financieras han concedido las operaciones y tipo de garantías que exige la SGR. Por último, analizamos la evolución en estos últimos años del número de empresas que están asociada al sector y que, por tanto, son beneficiarias del aval. El ritmo de crecimiento de la base societaria constituye otro buen indicador de la actividad desarrollada por estas sociedades.

\section{RESUMO}

Nós nos centramos neste artigo no estudo das garantias concedido pelas Sociedades diferentes de Garantia Recíproca, SGR que opera na Espanha; primeiro de um modo quantitativo, analisando a evolução do Risco Vivo variável, no período 1997-2002. Mais tarde nós levaremos a cabo um estudo qualitativo das garantias efetivas, enquanto ajudando a sete perspectivas diferentes: setores de atividade, tamanho das companhias, propósito das operações, termo de amortização, meia quantia, entidades financeiras concederam as operações e tipo de garantias que as demandas de SGR. Ultimamente, nós analisamos a evolução nestes últimos anos do número de companhias que são associadas ao setor e que, então, eles são os beneficiários da garantia. O ritmo de crescimento da base de associação constitui outro indicador bom da atividade desenvolvido por estas sociedades.

PALABRAS CLAVE: AVAL, PYME, RIESGO VIVO

KEY WORDS: GUARANTEE, OUTSTANDING COMMITMENTS, SME

\section{INTRODUCCIÓN}

Las Sociedades de Garantía Recíproca (en adelante, SGR) son sociedades constituidas por pequeñas y medianas empresas (en adelante, PYME) cuyo objeto fundamental es prestar las garantías necesarias para que sus socios-empresarios obtengan la financiación que necesitan para sus empresas. A estas sociedades acuden tanto las PYME que no tienen solvencia suficiente para obtener un crédito de las entidades financieras, pese a tener un proyecto de inversión rentable, como las que, teniendo garantías suficientes, buscan obtenerlo en mejores condiciones gracias al aval de la SGR.

Nos centramos en este artículo en el estudio de los avales concedidos por las diferentes SGR que operan en España; primero de una forma cuantitativa, analizando la evolución de la variable Riesgo Vivo, en el periodo 1997-2002. Dicha variable refleja el saldo pendiente de amortización al cierre de cada período por las operaciones avaladas así como por otras garantías prestadas y formalizadas por la sociedad. Con su estudio podremos observar la importancia de la actividad desarrollada por estas entidades y la evolución de la misma. 
Revista Eletrônica de Ciência Administrativa (RECADM) - ISSN 1677-7387

Faculdade Cenecista de Campo Largo - Coordenação do Curso de Administração

v. 3, n. 1, maio/2004 - http://revistas.facecla.com.br/index.php/recadm/

Posteriormente realizaremos un estudio cualitativo de los avales vigentes, atendiendo a siete perspectivas distintas, que nos permitirán responder a los siguientes interrogantes:

1. ¿A qué sectores de actividad pertenecen las empresas que han sido avaladas por las SGR?

2. ¿Qué tamaño tienen dichas empresas?

3. ¿Cuál es la finalidad de las operaciones que se han respaldado con el aval de estas entidades?

4. ¿Qué plazo de amortización tienen estas operaciones?

5. ¿Cuál es su importe medio?

6. ¿Qué entidades financieras han concedido las operaciones aceptando para ello el aval de las SGR?

7. ¿Qué tipo de garantías exige la SGR a la empresa a la que se le concede el aval?

Por último, nos detendremos en observar la evolución en estos últimos años del número de empresas que están asociada al sector y que, por tanto, son beneficiarias del aval. El ritmo de crecimiento de la base societaria constituye otro buen indicador de la actividad desarrollada por estas sociedades.

El análisis se realiza a partir de las cifras que nos ha suministrado la Confederación Española de Sociedades de Garantía Recíproca, CESGAR, y corresponden a los resultados obtenidos a 31 de Diciembre de 2002. Estos datos se ofrecen de forma agregada para todo el sector. No obstante, cuando hemos estimado conveniente, se ha realizado un análisis detallado por sociedad, haciendo uso de las memorias respectivas de cada una de ellas.

\section{EVOLUCIÓN DEL RIESGO VIVO}

En el Gráfico 1 se refleja el importe del Riesgo Vivo por avales y otras garantías mantenido por el sector en el periodo 1997-2002. De la observación del mismo, podemos extraer una primera conclusión: el sector ha experimentado importantes crecimientos estos últimos años, destacando 1997 y 1998, con incrementos superiores al 20\% respecto al año anterior. Las sociedades han pasado de los 1.137 millones de euros de Riesgo Vivo en 1997 hasta los niveles actuales; en sólo seis años ha crecido más del $117 \%$.

\section{EVOLUCIÓN DEL RIESGO VIVO}

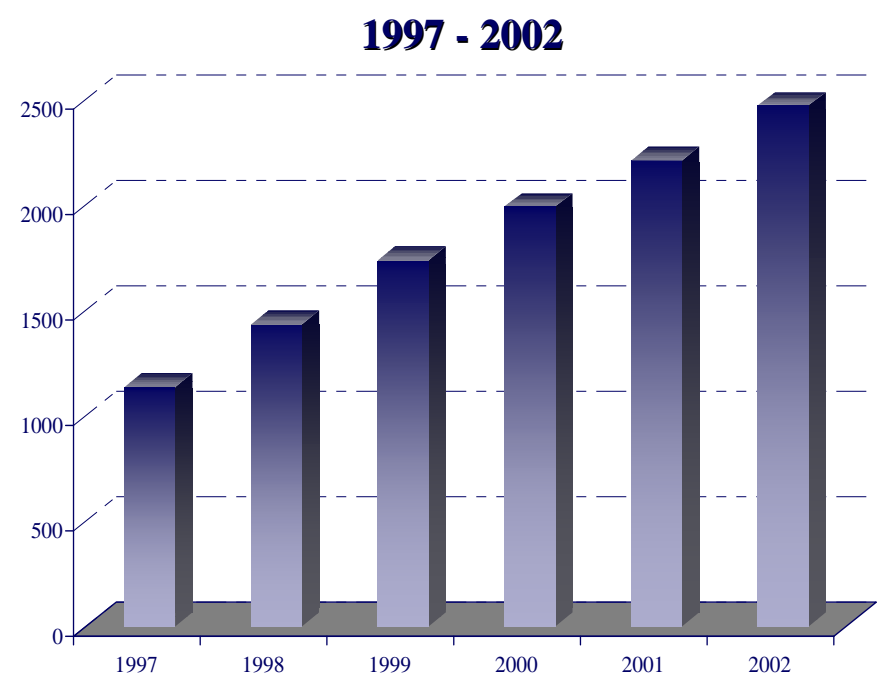

Gráfico 1

Fuente: CESGAR. Elaboración propia 


\section{Revista Eletrônica de Ciência Administrativa (RECADM) - ISSN 1677-7387 \\ Faculdade Cenecista de Campo Largo - Coordenação do Curso de Administração \\ v. 3, n. 1, maio/2004 - http://revistas.facecla.com.br/index.php/recadm/}

Sin embargo, hemos de señalar que no todas las sociedades muestran un comportamiento homogéneo, existiendo una gran concentración en el sector. Como podemos ver en la Tabla 1, si atendemos a la variable Riesgo Vivo, nos encontramos con tres grupos diferenciados:

- Un primer grupo compuesto por tres sociedades. Son las que operan habitualmente en la Comunidad Valenciana, SGR, SCV, en el País Vasco, ELKARGI, SGR y en Castilla y León, IBERAVAL, SGR. De los 2.468 millones de euros de avales que tienen en vigor todas las SGR del sector, 1.485 millones de euros corresponden a estas tres entidades, lo que supone el $60 \%$ de Riesgo Vivo total.

- Después podemos distinguir otro grupo de tamaño medio, con Riesgo Vivo entre 125 y 58 millones de euros: AFIGAL, SGR (Galicia), AVALMADRID, SGR (Comunidad de Madrid), AVALUNION, SGR (Andalucía), ISBA, SGR (Islas Baleares), SOGARPO, SGR (Galicia), SONAGAR, SGR (Navarra), SURAVAL y UNDEMUR, SGR (Murcia). Estas siete sociedades acaparan el $29,45 \%$ del total sectorial.

- $\quad$ Otro grupo de tamaño muy pequeño, estaría formado por aquellas con Riesgo Vivo inferior a los 39 millones de euros. Entre ellas destaca SOGARCA (Cantabria) por no alcanzar ni los 12 millones de euros en garantías vigentes. También a este grupo, pertenecen las tres sociedades de más reciente creación, en el año 1997, EXTRAVAL, SGR (Extremadura), FIANZAS Y SERVICIOS FINANCIEROS, SGR (Sociedad sectorial) y ONIARRI, SGR (País Vasco) que aún, por motivos obvios, no han alcanzado niveles importantes de riesgo pero que han crecido a gran ritmo desde el inicio de su actividad. Las restantes sociedades que aún no han sido mencionadas son SOGARTE, SGR ( Islas Canarias), ARAVAL, SGR (Aragón), ASTURGAR, SGR (Asturias), TRANSAVAL, SGR ( Sociedad sectorial), CREDIAVAL, SGR (Andalucía) y SOGAPYME, SGR (Islas Canarias). Estas once entidades no suman ni el $11 \%$ del Riesgo Vivo total.

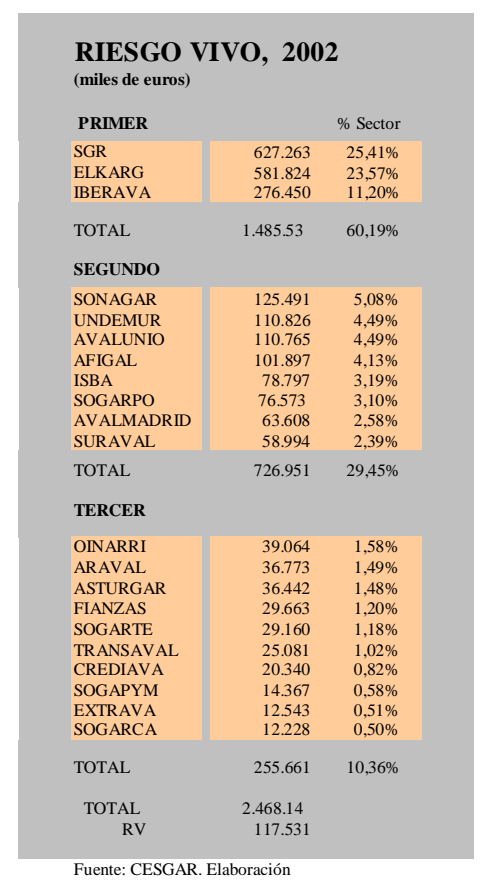




\section{Revista Eletrônica de Ciência Administrativa (RECADM) - ISSN 1677-7387 \\ Faculdade Cenecista de Campo Largo - Coordenação do Curso de Administração \\ v. 3, n. 1, maio/2004 - http://revistas.facecla.com.br/index.php/recadm/}

Por último, debemos señalar que aunque la actividad avalista es el objetivo más importante de estas sociedades no es el único. Actualmente, y cada año con mayor fuerza, desarrollan otras funciones complementarias a la prestación del aval. Nos estamos refiriendo a servicios de información que permiten a las PYME conocer los instrumentos financieros más adecuados a sus necesidades, a servicios de asesoramiento que proporcionan al empresario, analizando, evaluando y aconsejando sobre el proyecto de inversión que va a realizar y por último, servicios de formación financiera, mediante cursos, seminarios, etc... que estas entidades organizan. Además, las SGR pueden participar en el capital de sociedades cuyas actividades estén orientadas a favorecer a las PYME, una vez que hayan cubierto las reservas legalmente obligatorias.

\section{ANALISIS DESAGREGADO DE LOS AVALES}

En este apartado profundizamos en las características de los avales vigentes al término del año 2.002, desagregando el volumen de Riesgo Vivo bajo siete criterios diferenciados. Este análisis nos va a permitir conocer distintos aspectos del funcionamiento del sistema de garantías recíprocas. En primer lugar, dos características de las PYME que actualmente están avaladas por alguna SGR: los sectores a los que pertenecen y el tamaño empresarial medio. En segundo lugar, características específicas de las operaciones avaladas: la finalidad, el plazo de amortización y el importe medio de las mismas. Por último, dos datos relativos a la concesión del aval: las entidades financieras que finalmente aprueban las operaciones y ponen los recursos a disposición de las PYME y el tipo de garantías que exigen las SGR a sus asociadas para concederles el aval.

Por lo que se refiere al análisis sectorial, los datos de la distribución del riesgo vivo a 31 de Diciembre de 2002 según el sector de actividad, han sido recogidos en el Gráfico 2. De su análisis, se desprende un predominio claro del Sector Servicios, considerado en sentido amplio, que se mantiene en un $49 \%$ del total, y del que un 15\% corresponde al Comercio. Es seguido por el Sector Industrial, que representa un porcentaje en torno al 32\%. Por su parte, el Sector de la Construcción ocupa alrededor del $16 \%$ de la cartera de avales de las SGR, en gran parte debido a la prestación de avales técnicos. Por último, hemos de señalar el escaso porcentaje de operaciones concedidas en el Sector Primario, que sólo alcanza el 3\% del total.

\section{DISTRIBUCION SECTORIAL DEL} RIESGO VIVO

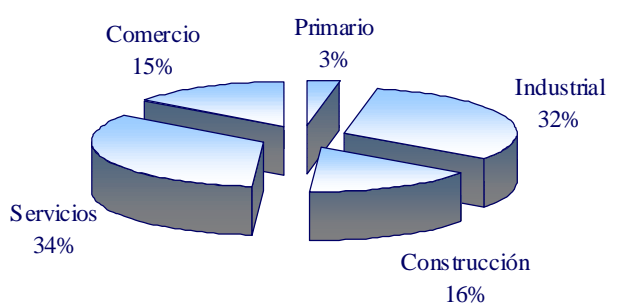

$16 \%$

Gráfico 2

Fuente: CESGAR. Elaboración propia

De otro lado, si analizamos el tamaño de las empresas que obtienen el aval y que, por tanto, están asociadas al sistema, podemos observar que el segmento empresarial al que dirige su actividad la SGR es la PYME y de forma más concreta la micropyme. En el Gráfico 3 se muestran los resultados de esta nueva distribución: más del $44 \%$ de las empresas avaladas tienen menos de 10 trabajadores y más del $64 \%$ no superan los 25 trabajadores. 
Revista Eletrônica de Ciência Administrativa (RECADM) - ISSN 1677-7387

Faculdade Cenecista de Campo Largo - Coordenação do Curso de Administração

v. 3, n. 1, maio/2004 - http://revistas.facecla.com.br/index.php/recadm/

\section{DISTRIBUCION DEL RIESGO VIVO POR TAMAÑO DE LA EMPRESA}

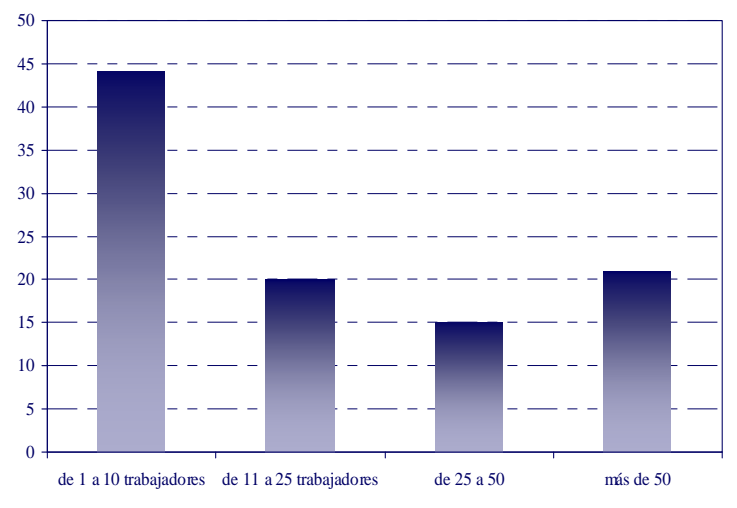

Gráfico 3

Fuente: CESGAR. Elaboración propia

Por otra parte, si atendemos a la finalidad de las operaciones avaladas, a la vista de los resultados obtenidos (Gráfico 4) podemos señalar que el porcentaje de avales que se destinan a Créditos para Nuevas Inversiones es mayoritario, situándose en torno al 59\%. Del resto, el 13\% va dirigido a operaciones de refinanciación de Circulante y el 24\% lo constituyen los Avales Técnicos. Estos últimos están otorgados para avalar la contratación de obras, servicios o suministros, así como la concurrencia a subastas ante las Administraciones Públicas, como garantías prestadas para la construcción de viviendas y, también, para garantizar obligaciones ante haciendas públicas, tribunales de justicia y otros organismos públicos.

\section{DISTRIBUCION DEL RIESGO VIVO} POR DESTINO DEL AVAL

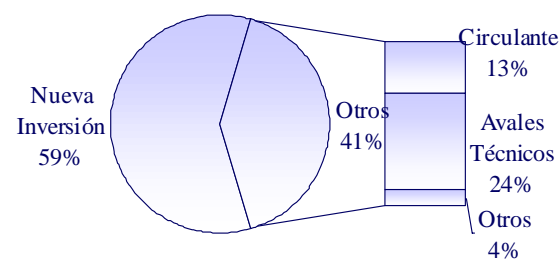

Gráfico 4

Fuente: CESGAR. Elaboración propia

Si atendemos al análisis del Riesgo Vivo según los plazos de vencimiento (Gráfico 5) podemos observar que el $90 \%$ del importe total de los avales en vigor tiene un vencimiento de más de 36 meses, es decir, están respaldando operaciones a largo plazo. Estos datos nos reflejan una de las funciones que cumplen las SGR, esto es, conseguir mejorar las condiciones de financiación, y entre ellas, el plazo de amortización de la operación. Es conocido que las PYME tienen dificultades de acceso a operaciones de plazos de devolución amplios, con el consiguiente perjuicio para el equilibrio 
Revista Eletrônica de Ciência Administrativa (RECADM) - ISSN 1677-7387

Faculdade Cenecista de Campo Largo - Coordenação do Curso de Administração

v. 3, n. 1, maio/2004 - http://revistas.facecla.com.br/index.php/recadm/

de sus estructuras económico-financieras. No obstante, conviene señalar, que estos datos hay que tomarlos con ciertas precauciones ya que para sus cálculos suelen incluirse los avales técnicos en vigor, a los que se les asigna vencimiento indeterminado.

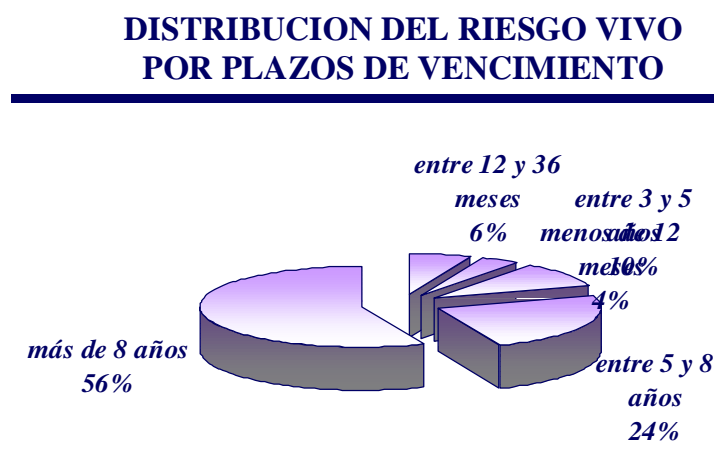

Gráfico 5

Fuente: CESGAR. Elaboración propia

En cuanto al importe medio de estos avales, el $79 \%$ del total del número de operaciones no superan los 66.000 euros. Como podemos apreciar en el Gráfico 6, es totalmente mayoritario este tipo de operaciones de pequeña cuantía, de forma coherente con el tipo de empresas con las que trabajan las SGR.

\section{DISTRIBUCION DEL RIESGO VIVO} POR IMPORTE AVALADO

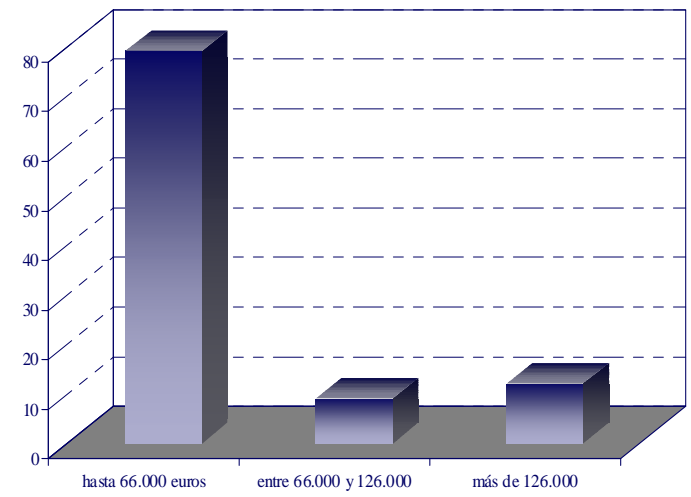

Gráfico 6

Fuente: CESGAR. Elaboración propia

Por otra parte, los datos referentes a la distribución de los avales en función de las entidades que conceden las operaciones (Gráfico 7) muestran un predominio absoluto de las Cajas de Ahorro que suponen el 38\% de los avales concedidos. La Banca Privada aparece en segundo lugar con el $22 \%$. Por último, señalar la importancia de los avales concedidos ante las Administraciones Públicas, el $22 \%$ y ante los Proveedores y Clientes, un $4 \%$. 
Revista Eletrônica de Ciência Administrativa (RECADM) - ISSN 1677-7387

Faculdade Cenecista de Campo Largo - Coordenação do Curso de Administração

v. 3, n. 1, maio/2004 - http://revistas.facecla.com.br/index.php/recadm/

DISTRIBUCION DEL RIESGO VIVO

POR ENTIDAD FINANCIERA

Gráfico 7

Fuente: CESGAR. Elaboración propia

Por último, la SGR, como contrapartida al aval otorgado a favor del empresario puede exigirle contragarantías, siendo válidas todas las admitidas en derecho y que resulten suficientes a juicio del avalista. El desglose según las contragarantías exigidas en las operaciones avaladas queda representado en el Gráfico 8 . En el mismo, podemos observar que el porcentaje de operaciones en las que se ha exigido Garantía Real alcanza el 36\%. Las operaciones a las que no se les ha exigido ningún tipo de garantías representan el $29 \%$ y señalamos que es destacable el incremento que se produce en estos últimos años en este tipo de operaciones. El 35\% restante lo constituyen las operaciones a las que se le ha exigido una Garantía Personal, siendo éstas las que han descendido en beneficio de las anteriores.

\section{DISTRIBUCION DEL RIESGO VIVO \\ POR GARANTIAS \\ COMPLEMENTARIAS}

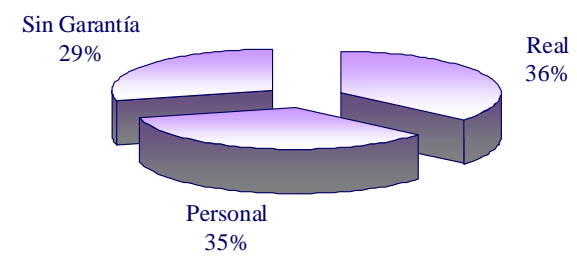

Gráfico 8

Fuente: CESGAR. Elaboración propia 
Revista Eletrônica de Ciência Administrativa (RECADM) - ISSN 1677-7387

Faculdade Cenecista de Campo Largo - Coordenação do Curso de Administração

v. 3, n. 1, maio/2004 - http://revistas.facecla.com.br/index.php/recadm/

\section{EVOLUCION DE LOS SOCIOS PARTÍCIPES}

Sólo cuando se adquiere la condición de Socio Partícipe puede solicitarse el aval de la sociedad. Por ello, es necesario que la PYME interesada se asocie al sistema participando en el capital social de alguna SGR. Deberá aportar además aquella documentación necesaria para que el equipo de analistas evalúe el riesgo de la operación. A este respecto, es importante destacar que los socios sólo responden de las deudas de la sociedad con el importe desembolsado por las cuotas sociales, que no se elevan a importes elevados.

Es precisamente el número de asociados al sistema uno de los indicadores que mejor puede mostrarnos si el sector de garantías recíprocas está creciendo y consolidándose dentro del Sistema Financiero Español. La evolución del mismo queda detallada en el Gráfico 9. En la actualidad, más de 63.000 empresas se están beneficiando de la labor de estas sociedades, bien porque se les ha facilitado el acceso al crédito, bien porque la empresa ha conseguido unas mejores condiciones, en plazo o coste, que las que hubiera obtenido sin la mediación de la SGR.

EVOLUCIÓN SOCIOS PARTICIPES

$1997-2002$



Gráfico 9

Fuente: CESGAR. Elaboración propia

Además, podemos observar el crecimiento continuado que desde 1997 se produce en el número de empresas asociadas. Esto es tanto más importante por cuanto que aquella empresa que ha finalizado su relación de garantía con la sociedad, puede solicitar el reembolso de sus cuotas y desligarse de la entidad. Aún así, en estos últimos años son más las que se incorporan que las que abandonan el sistema, más de 3.000 empresas adicionales cada año.

CESGAR, asociación que asume la coordinación, cooperación, defensa y representación de los intereses de las SGR en España recoge en sus informes anuales el impacto económico-social que produce la actividad de sus asociadas. En la última memoria, además de señalar que 63.793 empresas se han beneficiado del aval SGR, nos explica que los 8.214 millones de euros en avales concedidos han supuesto una inversión inducida de 10.055 millones de euros, afectando a 435.520 puestos de trabajo. Por último, indica que el apoyo de la administración se traduce en 6.000 euros por cada 35 PYME beneficiadas.

\section{CONCLUSIONES}

- El sector está creciendo a un ritmo importante. A la expansión del sistema contribuye, con seguridad, el marco legislativo actual, iniciado en 1994 y totalmente completado el pasado año. Tampoco podemos desligar este hecho del fuerte crecimiento económico de estos últimos años. 


\section{Revista Eletrônica de Ciência Administrativa (RECADM) - ISSN 1677-7387 \\ Faculdade Cenecista de Campo Largo - Coordenação do Curso de Administração \\ v. 3, n. 1, maio/2004 - http://revistas.facecla.com.br/index.php/recadm/}

Sin embargo, sería deseable que el reparto de las garantías fuera más homogéneo a través del territorio español. Existen comunidades que no cuentan con ninguna SGR con la desventaja que supone para las PYME en ellas localizadas. Es el caso de Castilla la Mancha y La Rioja, si bien esta última está atendida por las sociedades vascas (en Cataluña se ha creado en los últimos meses una SGR). Además, en otras comunidades autónomas aunque existe o existen SGR, presentan escasa actividad.

- Si analizamos el segmento empresarial que trabaja con estas entidades, nos encontramos que sectorialmente reproduce la distribución general de actividades en nuestro país. En cuanto al tamaño de las asociadas, un alto porcentaje son empresas de menos de 10 trabajadores. Estos datos también están conformes a las características del empresariado español, compuesto en más de un 95\% por PYME.

- En cuanto a las operaciones avaladas, recogemos dos datos positivos; primero, el que la mayoría de estas operaciones se destinen a financiar nuevas inversiones. En nuestra opinión, el papel de los avales técnicos, aunque importante si se ofrecen con mejores costes, no debe ser el objetivo de estas sociedades. Segundo, el alto plazo de vencimiento de las operaciones, reflejo de la búsqueda por parte de estas entidades de una mejor estructura financiera para las empresas asociadas, al permitirles acceder a la financiación a largo plazo, que tan reacias son las entidades crediticias a conceder.

Otra características de las operaciones, según los análisis realizados sobre el importe avalado, es su escaso volumen medio, en su mayoría de cuantía menor a 66.000 euros, coherente con el tipo de empresas a las que se le conceden los avales.

- Respecto al funcionamiento de la concesión del aval, destacar que un $29 \%$ de las operaciones se aprueban sin ningún tipo de contragarantías. Esto es importante siempre que sea significativo del apoyo a proyectos viables de emprendedores sin garantías. Por otra parte, son las Cajas de Ahorro las que, por el momento, mayor confianza depositan en el sistema de garantías y por ello son las que más trabajan en colaboración con las SGR aceptando su aval.

- La base societaria se amplía cada año y ello es indicativo de que el sistema de garantías recíprocas tiende a consolidar su posición en el sistema financiero español, en consonancia con otras economías del entorno europeo.

\section{BIBLIOGRAFÍA}

Confederación Española de Sociedades de Garantía Recíproca, CESGAR: memorias Sociedades de Garantía Recíproca: memorias 